Check for updates

Cite this: RSC Adv., 2017, 7, 39420

Received 23rd June 2017

Accepted 5th August 2017

DOI: $10.1039 / c 7 r a 06997 a$

rsc.li/rsc-advances

\section{Flexible piezocapacitive sensors based on wrinkled microstructures: toward low-cost fabrication of pressure sensors over large areas}

\author{
Seolhee Baek, $\dagger^{\mathrm{a}}$ Hayeong Jang, $\dagger^{\mathrm{a}}$ So Young Kim, ${ }^{\mathrm{c}}$ Heejeong Jeong, ${ }^{a}$ Singu Han, ${ }^{a}$ \\ Yunseok Jang, ${ }^{\star b}$ Do Hwan Kim (iD *c and Hwa Sung Lee (iD) *a
}

\begin{abstract}
Flexible pressure sensors are a key component of electronic skin (e-skin) for use in future applications ranging from human healthcare monitoring to robotic skins and environmental risk detection. Here, we demonstrated the development of a highly sensitive, simple, and low-cost capacitive pressure sensor, which acted as a flexible capacitive dielectric, based on a microstructured elastomeric template that could be fabricated over a large area. To achieve this goal, the dielectric template was prepared simply by stretching and releasing a flexible Ecoflex film to produce wrinkled surface microstructures with a feature size on the order of tens of micrometers. The effects of the wrinkled surface microstructure on the sensing performance were systematically investigated by comparing the nonwrinkled film, one-side wrinkled film, and double-side wrinkled film. The response and release times of the double-side wrinkled pressure sensor were improved by $42 \%$ and $25 \%$ in comparison with the values obtained from the unwrinkled case, respectively. These results showed that the introduction of wrinkled surface microstructures to the elastomeric template efficiently enhanced the pressure sensor performance. We also demonstrated that our sensor could be used to detect a variety of changes in the surroundings, such as variations in the angle of a stimulus, object loading/unloading, or an exhaled breath.
\end{abstract}

\section{Introduction}

Human-adaptive stretchable electronic skin (e-skin) has recently attracted a great deal of attention due to its ability to sense stimulus changes. ${ }^{1-5}$ This functionality is useful in wearable health monitoring, sensitive tactile information displays, prosthetics, and multifunctional robot skin applications. ${ }^{1-6}$ Remarkable advances in noninvasive human body-monitoring devices have been made using ultrathin and ultralight layers with electrically active matrixes that can adhere to the surfaces of skin and other objects. ${ }^{2}$ However, the human body consists of many nonflat surfaces with fine topology, thus ultraconformal contact onto irregular, nonflat, and coarse structures remains a challenge for realizing real-time diagnoses. A variety of highly sensitive pressure sensors and integrated multifunctional devices using stretchable and flexible networks have been tested in an effort to overcome the problems mentioned above., ${ }^{2,4,6-8}$

\footnotetext{
${ }^{a}$ Department of Chemical and Biological Engineering, Hanbat National University, Daejeon 34158, Korea.E-mail: hlee@hanbat.ac.kr

${ }^{b}$ Advanced Manufacturing Systems Research Division, Korea Institute of Machinery and Materials KIMM, Daejeon 34103, Korea. E-mail: yjang@kimm.re.kr

${ }^{{ }^{2} D e p a r t m e n t ~ o f ~ O r g a n i c ~ M a t e r i a l s ~ a n d ~ F i b e r ~ E n g i n e e r i n g, ~ S o o n g s i l ~ U n i v e r s i t y, ~ S e o u l ~}$ 06978, Korea.E-mail:dohwan@ssu.ac.kr

$\dagger$ S. Baek and H. Jang contributed equally to this work.
}

The functionalities of e-skin capable of detecting human physical data can be improved through the rational design of sensing materials and device architectures., ${ }^{4,6,9-12}$ Polydimethylsiloxane (PDMS) is the most popular elastic material used to integrate sensitive nanomaterials into flexible e-skin applications due to its excellent elasticity and biocompatibility. Microstructured elastomeric films are a key element of e-skin devices, providing better sensitivities and faster response times compared to unstructured thin films., ${ }^{40-12}$ Application of an external stimulus, especially pressure, to microstructured elastomeric features induces elastic deformation that store and release energy reversibly. The reversible material response minimizes problems associated with the viscoelastic behaviors of an elastomer. The compression of unstructured elastomer templates increases the relaxation time, and a lack of deformable surface structures reduces the responsiveness of the material to an external pressure load. Uniformly microstructured elastomeric templates are usually fabricated using patterned mold transfer or photolithography techniques. ${ }^{11,13-15}$ These fabrication processes control the geometry and shape of the elastomeric template micro-features by forming pyramids, cubes, lines, hemispheres, columns, etc.

These processes are expensive, however, require multiple steps (spin-coating, lithography, and etching), and the large-area integration of microstructures into a template surface tends to display poor reproducibility. Silk-molded structures were recently 
introduced as a simple method for fabricating large-area patterned PDMS thin films with a uniform microstructure for monitoring human physiological signals. ${ }^{9}$ The silk-molded structures were applied to a microstructured electrode template, rather than an elastomeric dielectric. A sensor device prepared using patterned carbon nanotube/PDMS films demonstrated an ultrahigh sensitivity for the detection of minute forces, with a detection limit as low as $0.6 \mathrm{~Pa}$. This approach provided a facile method for fabricating conformal sensing structures over large areas for the collection of physical data, such as the pulse, changes in skin contact, and body motions.

Here, we describe a simple and low-cost process for fabricating a large-area microstructured elastomeric template that acted as a flexible capacitive pressure sensor. To achieve this goal, a soft Ecoflex dielectric film was pre-strained in a first step. The Ecoflex surface was then treated with ultraviolet-ozone (UVO) exposure to form a stiff silicate layer on the surface. ${ }^{\mathbf{1 6 , 1 7}}$ The strain on the Ecoflex film was then relaxed, resulting in the formation of a microstructured wrinkled surface with features on the order of tens of micrometers. The Ecoflex template with wrinkled microstructure was sensitively deformed to permit the reversible storage and release of energy generated under an external pressure. Finally, a Au-coated silicon wafer (hard) and a PDMS (soft) later were positioned on the top and bottom of the wrinkled Ecoflex template to form electrodes. The effect of the wrinkled structure on the flexible pressure sensor was explored by systematically comparing three films: nonwrinkled film, oneside wrinkled film, and double-side wrinkled film. The constructed flexible pressure sensor was characterized by its mechanical capacitance response as a function of the applied loading value. The wrinkled pressure sensor displayed a high sensitivity, a mechanically rapid responsivity (pressing and releasing), good durability, and performance repeatability. In addition, the wrinkled pressure sensor effectively detected the degree to which a book was opened, the presence of an object, and the pressure differential of an exhaled breath the fabrication processes of pressure sensor were easy and compatible with conventional microfabrication technology, which permits scalable production at a low unit cost.

\section{Experimental section}

\section{Fabrication of the wrinkled Ecoflex pressure sensor}

The Ecoflex template was prepared by mixing a prepolymer and a curing agent (0030, Smooth-On Inc.) in a weight ratio of $10: 1$, followed by curing at $60{ }^{\circ} \mathrm{C}$ for $1 \mathrm{~h}$. The fully cured Ecoflex film ( $\sim 1 \mathrm{~mm}$ in thickness) was uniaxially stretched and fixed to the home-built stretching stage. The film stretched through 200\% of its original length was placed in a UVO chamber (AH1700, 28 $\mathrm{mW} \mathrm{cm}^{-2}$, Ahtech LTS Co., Ltd.) for $30 \mathrm{~min}$ to form a hard silicate surface layer. After UVO treatment, the prestrain was removed, resulting in a wrinkled structure on the Ecoflex template surface. The templates were then stored in a vacuum desiccator over 1 day. Gold electrodes were fabricated by thermally evaporating a $100 \mathrm{~nm}$ thick Au layer onto the polydimethylsiloxane (PDMS) and silicon wafer to form soft and hard electrodes, respectively. Finally, the two electrodes were positioned above and below the template.

\section{Characterization of the wrinkled pressure sensor}

The response of the capacitive wrinkled pressure sensor against multiple stimuli was assessed by measuring the resistance and capacitance using an Agilent 4284A Precision LCR meter. The pressure or flexion sensing capabilities of the sensor were characterized using a home-built sensor probe station outfitted with a programmable $x y$ - and $z$-axis moving stage having $50 \mathrm{~nm}$ resolution, a force gauge (Mark-10, Electromatic Equip't Co., Inc.), and a bending machine, all interfaced through a computer. The force gauge measured the applied load, and the corresponding pressure was calculated by dividing the probe with the area.

\section{Structural analysis of the wrinkled pressure sensor}

The morphologies of the wrinkled Ecoflex surface were characterized using optical microscopy (OM, Eclipse 80i, Nikon) and field-emission scanning electron microscopy (SEM, Hitachi S-4200). The thicknesses of the Ecoflex templates were measured using both SEM and an alpha-step profilometer (Dektak 150, Veeco). The surface energies of the Ecoflex and UVO-treated Ecoflex were assessed by measuring the contact angles of distilled water and diiodomethane as probe liquids on the surface, using a contact angle analyzer (Phoenix 300A, SEO Co., Inc.). The $\gamma_{\mathrm{s}}$ values were calculated by fitting the following geometric mean equation to the measured values of the contact angles:

$$
1+\cos \theta=\frac{2\left(\gamma_{\mathrm{s}}^{\mathrm{d}}\right)^{1 / 2}\left(\gamma_{\mathrm{lv}}^{\mathrm{d}}\right)^{1 / 2}}{\gamma_{\mathrm{lv}}}+\frac{2\left(\gamma_{\mathrm{s}}^{\mathrm{p}}\right)^{1 / 2}\left(\gamma_{\mathrm{lv}}^{\mathrm{p}}\right)^{1 / 2}}{\gamma_{\mathrm{lv}}},
$$

where $\gamma_{\mathrm{s}}$ and $\gamma_{\mathrm{lv}}$ are the surface energies of the dielectric surface and the probe liquid, respectively, and the superscripts $d$ and $p$ refer to the dispersive and polar (nondispersive) components of the surface energy, respectively. The results were summarized in Table 1.

\section{Results and discussion}

Fig. 1a illustrates the procedure used to fabricate the pressure sensors using a wrinkled elastomeric template. The wrinkled template was fabricated by mechanically stretching the crosslinked Ecoflex film, followed by UVO exposure and strain release. As the strain was released, periodic wavy microstructures perpendicular to the pre-strain direction were generated on the Ecoflex surface. Mismatch between the elastic moduli of the Ecoflex bulk and silicate surface regions produced wrinkles. ${ }^{16-19}$ Typical features of the cross-sectional images of the nonwrinkled, one-side wrinkled, and double-side wrinkled Ecoflex templates prepared in our systems were clearly observed in the FE-SEM images shown in Fig. 1b-d. The top-view inset images of each sample revealed that the wrinkled Ecoflex templates were constructed with plenty of parallel wrinkles over a large area, indicating that the surface wrinkles were uniform. The one-side wrinkled film was characterized by a wrinkle 
Table 1 Summary of the contact angles and the surface energies of bare and UVO-treated Ecoflex templates

\begin{tabular}{|c|c|c|c|c|c|}
\hline & \multicolumn{2}{|c|}{ Contact angle $\left({ }^{\circ}\right)$} & \multicolumn{3}{|c|}{ Surface energy $\left(\mathrm{mJ} \mathrm{m}^{-2}\right)$} \\
\hline & Water & Diiodomethane & Polar term & Dispersion term & Total \\
\hline UVO-treated Ecoflex & $73 \pm 2$ & $46 \pm 1$ & 8.5 & 30.8 & 39.3 \\
\hline
\end{tabular}

(a)

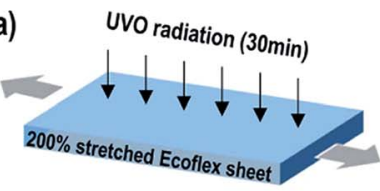
Releasing
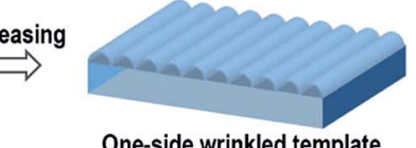

One-side wrinkled template

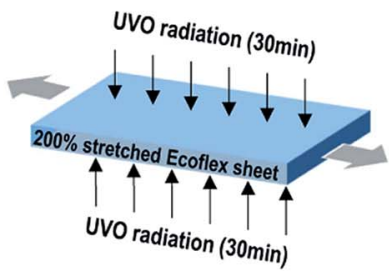

(b)
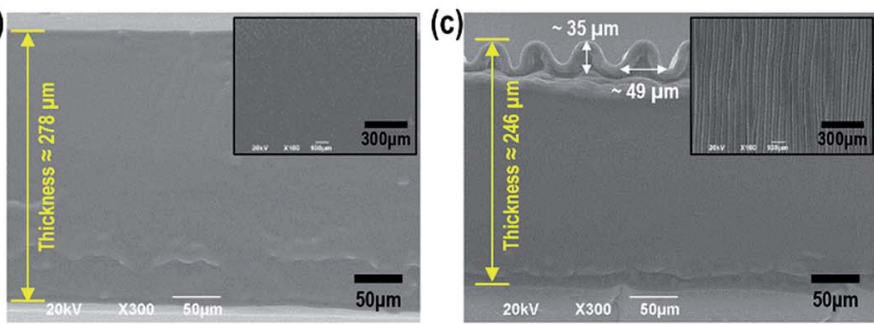

Releasing

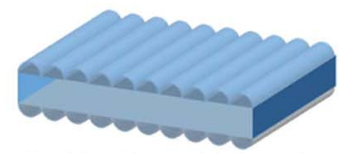

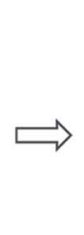

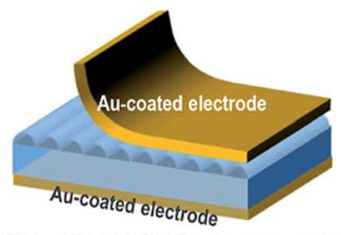

One-side wrinkled pressure sensor
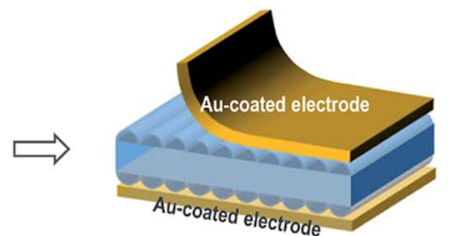

Double-side wrinkled pressure sensor

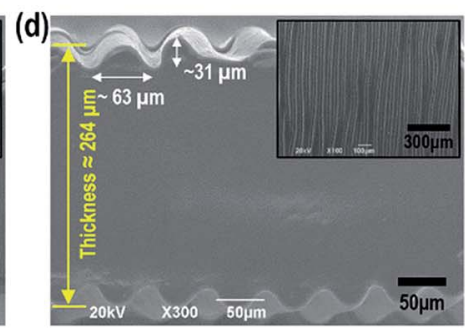

Fig. 1 (a) Schematic illustration of the procedure used to fabricate the wrinkled pressure sensors. (b-d) SEM images of the nonwrinkled, one-side wrinkled, and double-side wrinkled Ecoflex template films. The inset images show the top views of the Ecoflex template surfaces.

wavelength and amplitude of 49 and $35 \mu \mathrm{m}$, respectively. On the other hand, the double-side film displayed a slightly longer wavelength of $63 \mu \mathrm{m}$ and a lower amplitude of $31 \mu \mathrm{m}$ in the wrinkled microstructures. These results could be explained by the decrease in the elastic bulk region and the increase in the stiff surface region as a result of the double-side exposure to UVO. In addition, the stiff layers produced by the silicate on the UVO-treated Ecoflex can be obviously observed in Fig. 1c and d. (Consider the lack of image contrast in the double-side case of Fig. 1d). The patterned microstructures with numerous wrinkles efficiently increased the density of contact sites available to the pressure sensing device, thereby improving the degree of template deformation. The good sensitivity of our system was shown to be advantageous for applications.

The typical features of the wrinkled Ecoflex template situated between two electrodes are shown with clear optical contrast in the OM image presented in Fig. 2a. The wrinkled structures were uniformly distributed over a large area in the perpendicular direction of the strain, as explained in Fig. 1. The pressure sensing capability of the wrinkled pressure sensor was examined by applying a normal force to the unit sensing cell using a custom-built sensor probe station. Fig. $2 \mathrm{~b}$ and $\mathrm{c}$ show the capacitance change ratio $\left(\Delta C / C_{0}\right)$ as a function of the normal pressure applied to the wrinkled structure of the elastomeric templates using silicon wafer (hard) and PDMS (soft) electrodes, respectively. The intrinsic response characteristics of the wrinkled Ecoflex templates to the external pressure were confirmed using the hard electrode, as shown in Fig. 2b. The relative $\Delta C / C_{0}$ ratio obtained from the nonwrinkled, one-side wrinkled, and double-side wrinkled pressure sensors in Fig. $2 \mathrm{~b}$ revealed a quasi-bilinear dependence on the pressure at each pressure regime (low pressure regime: $<1.0 \mathrm{kPa}$ and high pressure regime: $>8.0 \mathrm{kPa}$ ) due to the incompressibility of the Ecoflex dielectric. ${ }^{20,21}$ Pressure sensitivity, $S$, defined as the slope of the traces, is commonly used to evaluate the performance of a pressure sensor. ${ }^{20,23,24}$ At pressures below $1.0 \mathrm{kPa}$ or above $8.0 \mathrm{kPa}$, the sensitivities of the nonwrinkled sensor were $4.8 \times 10^{-6}$ and $5.2 \times 10^{-6} \mathrm{kPa}^{-1}$, respectively. The pressure sensitivities increased with the addition of the wrinkled structures, to $3.4 \times 10^{-5}$ and $0.013 \mathrm{kPa}^{-1}$ for the one-side wrinkled and double-side wrinkled cases at low pressures, respectively. The results obtained from the wrinkled pressure sensors, 

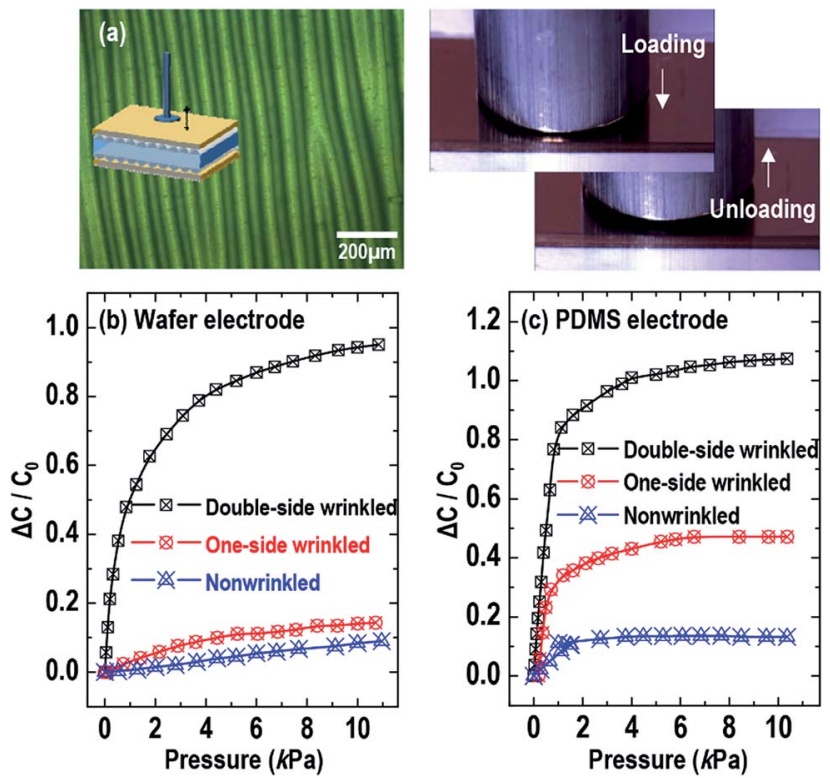

Fig. 2 (a) Optical microscopy image (left) of the wrinkled Ecoflex template, and the captured CCD images (right) during pressure loading and unloading using a custom-built sensor probe station. The relative capacitance change $\left(\Delta C / C_{0}\right)$ versus the normal pressure as a function of the wrinkled structures of the Ecoflex template, using (b) a silicon wafer (hard) and (c) PMDS (soft) electrodes coated with Au.

especially the double-side wrinkled film, in the high-pressure regime were higher than those obtained from the nonwrinkled pressure sensor.

The pressure sensing capabilities of our sensors were assessed using flexible PDMS electrodes. As shown in Fig. 2c, the pressure sensitivities increased in the order of nonwrinkled, one-side wrinkled, and double-side wrinkled Ecoflex pressure sensors. The capacitance change followed the same trend. The pressure sensitivities of our devices are summarized in Table 2. Note that the degree of the response or sensitivity to the external pressure improved significantly upon introduction of the wrinkled structure into the elastomeric template. For a given elastomeric template structure, some pressure sensitivity measurements were higher using the PDMS electrodes, whereas others were higher using the wafer electrodes. The differences between the two values did not follow a specific trend based on the template structure. These results were attributed not to the template structure, but to the absorption of some pressure by the PDMS electrode.
The operating reliabilities and response times of the wrinkled pressure sensors were assessed for the nonwrinkled, oneside wrinkled, or double-side wrinkled sensors. The device durability was obtained by measuring the capacitance of the device over 40 repeated load/unload cycles with an applied pressure of $5 \mathrm{kPa}$ (Fig. $3 \mathrm{a}-\mathrm{c}$ ). The pressure sensitivity of the elastomeric Ecoflex dielectric increased with increasing wrinkled surface structure, providing capacitance change ratios $(\Delta C)$ $C_{0}$ ) of $0.21,0.44$, and 0.97 for the nonwrinkled, one-side wrinkled, and double-side wrinkled sensors, respectively. These results could be explained in terms of the easy transformation of the wrinkled microstructures compared to the resistance to transformation by the flat structure of the Ecoflex dielectric surface. The typical response times for the nonwrinkled, oneside wrinkled, and double-side wrinkled sensors were $1.0 \mathrm{~s}$, $907 \mathrm{~ms}$, and $578 \mathrm{~ms}$, respectively (Fig. 3d-f). The typical release times were $1.04 \mathrm{~s}, 821 \mathrm{~ms}$, and $782 \mathrm{~ms}$, respectively (Fig. 3d-f). These results revealed that the wrinkled microstructures of the elastomeric template enhanced the response speed to the external stimulus because the wrinkled micro-features on the Ecoflex template deformed to a greater extent, thereby storing and releasing energy reversibly, which minimized problems due to the viscoelastic behavior of the elastomeric Ecoflex.

The step-by-step repeatable performances of the doublewrinkled pressure sensors over several cycles are shown in Fig. 4a. Excellent operational stability and reversibility were demonstrated over a 50 cycle run with a $0-8 \mathrm{kPa}$ force step. The force step and the cycle-to-cycle similarities are highlighted in Fig. $4 \mathrm{~b}$ and c. As shown in the figures, the continuous application of a pressure to the capacitive sensor decreased the thickness of the Ecoflex template between the two PDMS electrodes, which increased the capacitance. Despite the enhanced sensitivity obtained from the lower-modulus Ecoflex template, however, the value of the $\Delta C / C_{0}$ sensitivity gradually decreased during the step-by-step pressure increase. These results may indicate deformation saturation. Note that an increase in the sensitivity to each pressure step in our system was also evident in the sensors prepared with the soft PDMS electrodes. These results revealed that the introduction of a wrinkled surface microstructure to the elastomeric template clearly enhanced the performances of the pressure sensors. Recently, several studies have examined the increase in the operational range or linearity of the relationship between the sensor output and the applied pressure. ${ }^{6-10,22-31}$ From this standpoint, our sensor provided excellent sensitivity and reversibility, even though these performances have not yet surpassed the current best value.

Table 2 Pressure sensitivity S (or G-factor), defined as the slope of the trace used to evaluate the sensor performance. The values were extracted from a linear fit of the relative capacitance change $\left(\Delta C / C_{0}\right)$ as a function of the applied pressure

\begin{tabular}{llllll}
\hline & \multicolumn{2}{l}{ Wafer $($ hard) electrode } & & \multicolumn{2}{l}{ PDMS (soft) electrode } \\
\cline { 2 - 3 } & Low pressure $(<1 \mathrm{kPa})$ & High pressure $(>8 \mathrm{kPa})$ & & Low pressure $(<1 \mathrm{kPa})$ & High pressure $(>8 \mathrm{kPa})$ \\
\hline$G$-factor $\left(\mathrm{kPa}^{-1}\right.$, nonwrinkled) & $4.8 \times 10^{-6}$ & $5.2 \times 10^{-6}$ & & $7.6 \times 10^{-5}$ & $8.0 \times 10^{-7}$ \\
$G$-factor $\left(\mathrm{kPa}^{-1}\right.$, one-side) & $3.4 \times 10^{-5}$ & $5.8 \times 10^{-6}$ & & $8.8 \times 10^{-4}$ & $1.3 \times 10^{-6}$ \\
$G$-factor $\left(\mathrm{kPa}^{-1}\right.$, double-side) & 0.013 & $1.1 \times 10^{-5}$ & & 0.0012 & $4.2 \times 10^{-6}$
\end{tabular}



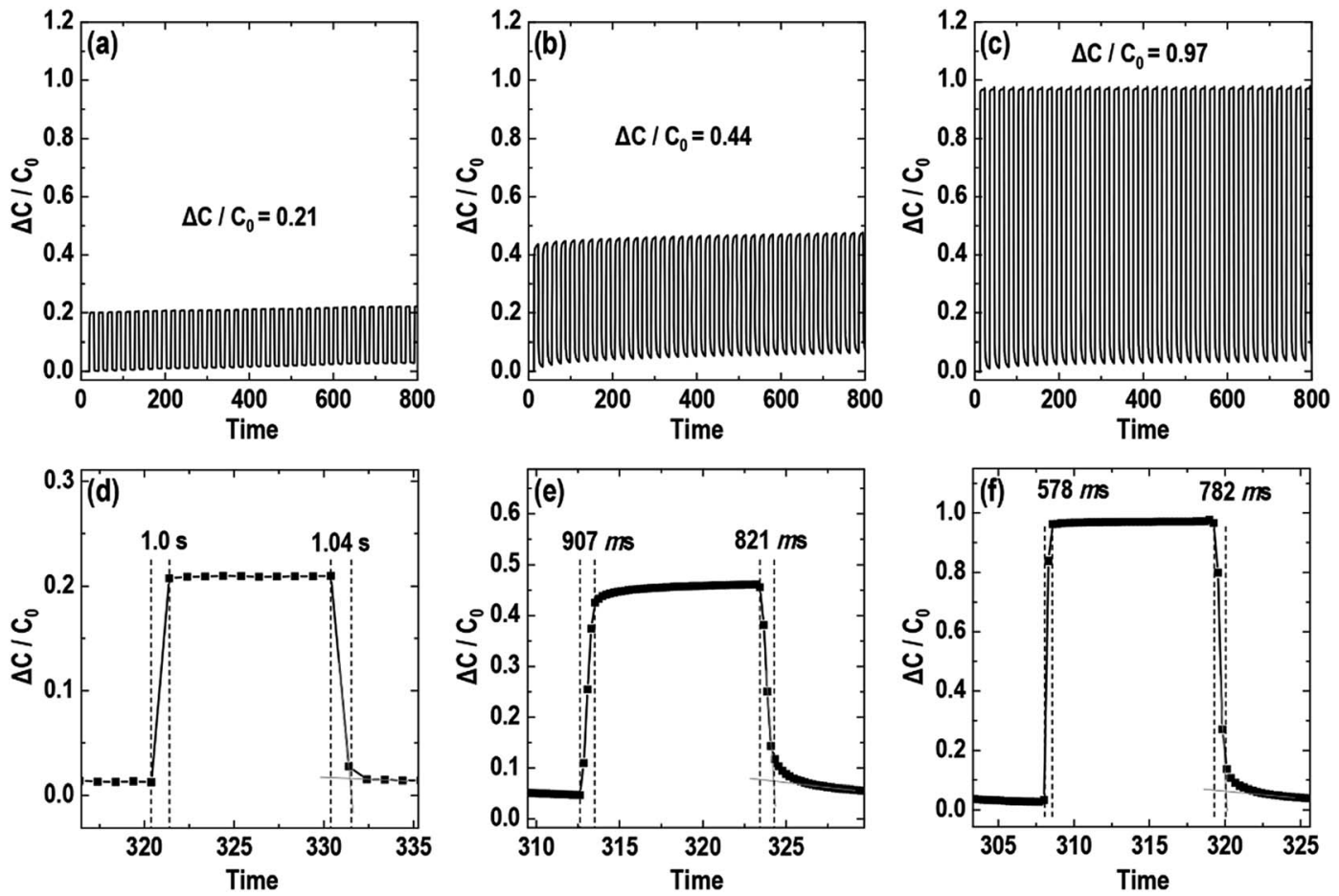

Fig. 3 Durability test under an applied pressure of $5 \mathrm{kPa}$ over more than 40 cycles for the (a) nonwrinkled, (b) one-side wrinkled, and (c) doubleside wrinkled pressure sensors. Pressure responses during single cycles, showing the response and releasing times for the (d) nonwrinkled, (e) one-side wrinkled, and (f) double-side wrinkled pressure sensors.

(a)

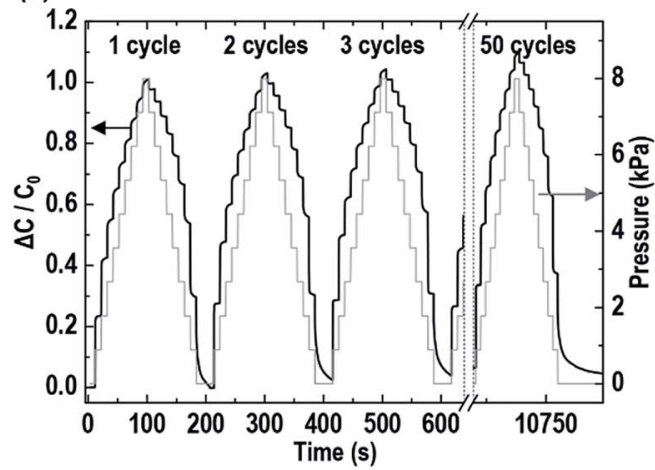

(b)

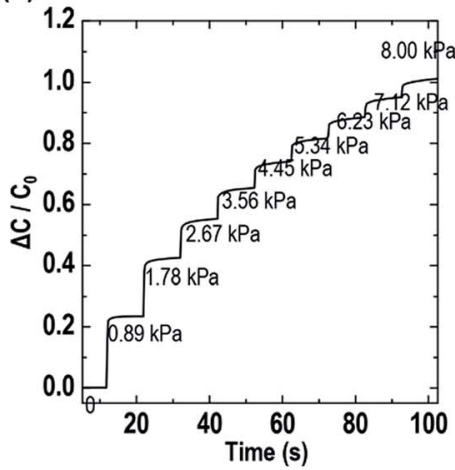

(c)

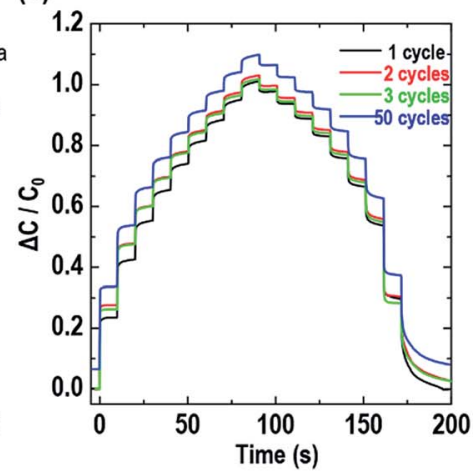

Fig. 4 (a) Step-by-step repeatability test as measured by $\Delta C / C_{0}$ over repeated loading/unloading cycles. (b) Force step used in the step-by-step repeatability test. (c) Overlapping graphs of the step-by-step repeatability test.

We examined the wrinkled pressure sensor for use as a folding-type sensing device fixed to the inner binding of a book (Fig. 5a). The sensitivities of the sensors to the book cover bending angle were investigated by measuring the capacitance values at different angles $\theta$, by closing the book at $40^{\circ}, 80^{\circ}$, and $120^{\circ}$ angles, in a step-wise manner (each angle was held over several seconds), followed by opening the book gradually at $120^{\circ}$, $80^{\circ}$, and $40^{\circ}$ angles. The change in the relative capacitance increased upon closing the book from $40^{\circ}$ to $120^{\circ}$ due to the compression induced by the tensile strain; whereas the change in the relative capacitance decreased upon opening the book from $120^{\circ}$ to $40^{\circ}$ due to the release of the compressive strain, as shown in the inset images of Fig. 5a. The folding-type sensors responded rapidly at each angle change, and the resistance remained constant for a given angle. The folding-type sensor using a wrinkled pressure sensor permits measurements of the changes in robotic joint angles, suggesting that the sensors could replace encoders. These results indicate that our sensors are attractive for applications in robotics and in detecting the open/closed state of a door in a multifunctional intelligent room system. The wrinkled pressure sensor could be used to detect the dynamic loading and unloading pressures of a small toy $150 \mathrm{mg}$ 

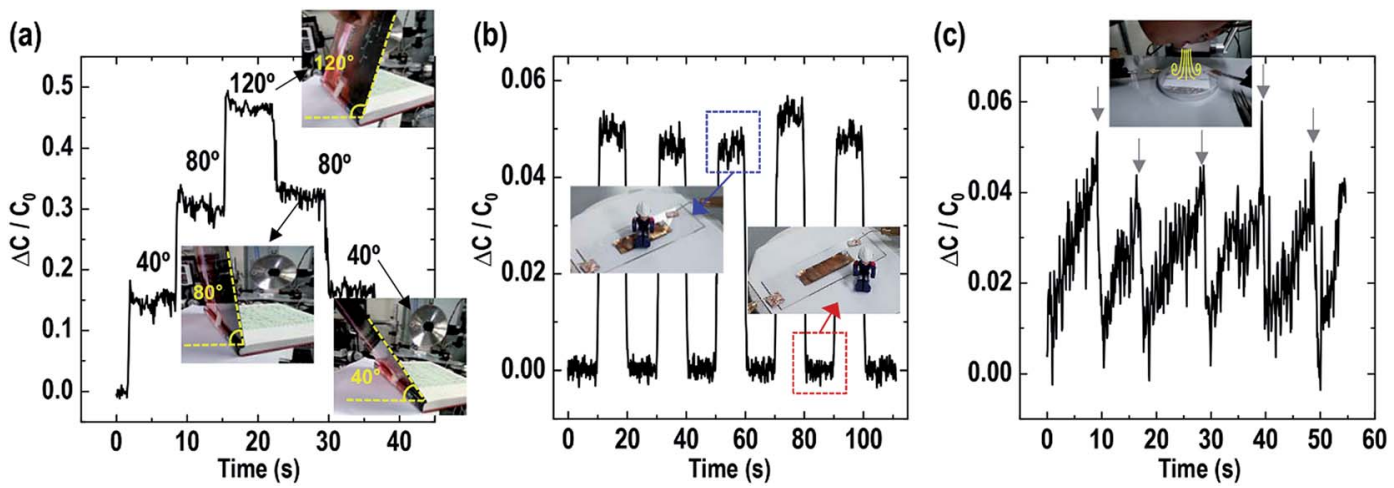

Fig. 5 Detection of three types of mechanical forces. (a) Capacitance response plot obtained from the wrinkled pressure sensor under closed (left side) and opened (right side) book states. (b) Capacitance response plot for the dynamic loading and unloading cycles of a small toy (150 mg). (c) Capacitance response plot measured in front of the mouth during an exhale. The photographs in the inset of each panel demonstrate the actions labelled.

in weight (Fig. 5b). We also observed a change in the capacitance during an exhale, as shown in Fig. 5c. High signal-to-noise ratios were obtained from all three types of pressure measurement, further demonstrating the highly sensitive capabilities of our wrinkled pressure sensors. These results suggest that our pressure sensor based on a wrinkled elastomeric template, fabricated using a simple and cheap process, constitutes an effective device structure for enabling e-skins capable of detecting applied pressures (or contact forces).

\section{Conclusions}

In summary, we developed a simple method for efficiently fabricating sensitive pressure sensors based on a micropatterned elastomeric template. A wrinkled microstructure Ecoflex surface was designed for use as a flexible capacitive dielectric that could sensitively deform. The effect of the wrinkled structures on the sensor performance was systematically investigated by fabricating a nonwrinkled microstructure, a one-side wrinkled microstructure, and a double-side wrinkled microstructure. Our pressure sensor constructed using the wrinkled Ecoflex template between the upper and bottom electrodes demonstrated a high sensitivity for the detection of minute external pressures with a fast response time, good stability, and good repeatability. The typical response and releasing times improved dramatically in the order of the nonwrinkled, one-side wrinkled, and double-side wrinkled pressure sensors, providing response times of $1.0 \mathrm{~s}, 907 \mathrm{~ms}$, and $578 \mathrm{~ms}$ and release times of $1.04 \mathrm{~s}, 821 \mathrm{~ms}$, and $782 \mathrm{~ms}$, respectively. These results revealed that the wrinkled surface microstructure on the elastomeric template efficiently enhanced the pressure sensor performance, even though our pressure sensor performance has not yet surpassed the current best value. Finally, the wrinkled pressure sensor was successfully used to monitor the angle of a book cover, the loading/ unloading of a small toy, and the pressure changes occurring at a mouth during an exhale. The pressure sensors developed here show promise in cost-efficient simple-to-manufacture wearable electronics for the prevention of potential risk factors from the external surroundings.

\section{Conflicts of interest}

There are no conflicts to declare.

\section{Acknowledgements}

This work was supported by Basic Science Research Program through the National Research Foundation of Korea (NRF) funded by the Ministry of Education (NRF-2016R1D1A1B03936094). Also, this work was supported by the Center for Advanced SoftElectronics under the Global Frontier Project (NRF2014M3A6A5060932) and the Basic Science Research Program (NRF-2017R1A2B4012819) of the National Research Foundation of Korea (NRF) funded by the Ministry of Science, ICT and Future Planning.

\section{References}

1 B. C.-K. Tee, A. Chortos, R. R. Dunn, G. Schwartz, E. Eason and Z. Bao, Adv. Funct. Mater., 2014, 24, 5427-5434.

2 M. L. Hammock, A. Chortos, B. C.-K. Tee, J. B.-H. Tok and Z. Bao, Adv. Mater., 2013, 25, 5997-6038.

3 J. Park, Y. Lee, J. Hong, M. Ha, Y.-D. Jung, H. Lim, S. Y. Kim and H. Ko, ACS Nano, 2014, 8, 4689-4697.

4 D. H. Ho, Q. Sun, S. Y. Kim, J. T. Han, D. H. Kim and J. H. Cho, Adv. Mater., 2016, 28, 2601-2608.

5 J. Cui, B. Zhang, J. Duan, H. Guo and J. Tang, Sensors, 2016, 16, 2131.

6 H. Tao, J. M. Kainerstorfer, S. M. Siebert, E. M. Pritchard, A. Sassaroli, B. J. B. Panilaitis, M. A. Brenckle, J. J. Amsden, J. Levitt, S. Fantini, D. L. Kaplan and F. G. Omenetto, Proc. Natl. Acad. Sci. U. S. A., 2012, 109, 19584-19589.

7 T. Takahashi, K. Takei, A. G. Gillies, R. S. Fearing and A. Javey, Nano Lett., 2011, 11, 5408-5413.

8 D. H. Kim, N. S. Lu, R. Ma, Y. S. Kim, R. H. Kim, S. D. Wang, J. Wu, S. M. Won, H. Tao, A. Islam, K. J. Yu, T. I. Kim, R. Chowdhury, M. Ying, L. Z. Xu, M. Li, H. J. Chung, H. Keum, M. McCormick, P. Liu, Y. W. Zhang, F. G. Omenetto, Y. G. Huang, T. Coleman and J. A. Rogers, Science, 2011, 333, 838-843. 
9 X. Wang, Y. Gu, Z. Xiong, Z. Cui and T. Zhang, Adv. Mater., 2014, 26, 1336-1342.

10 T. Someya, Y. Kato, T. Sekitani, S. Iba, Y. Noguchi, Y. Murase, H. Kawaguchi and T. Sakurai, Proc. Natl. Acad. Sci. U. S. A., 2005, 102, 12321-12325.

11 S. Mannsfeld, B. Tee, R. Stoltenberg, C. Chen, S. Barman, B. Muir, A. Sokolov, C. Reese and Z. Bao, Nat. Mater., 2010, 9, 859-864.

12 F. Fan, L. Lin, G. Zhu, W. Wu, R. Zhang and Z. L. Wang, Nano Lett., 2012, 12, 3109-3114.

13 G. Y. Bae, S. W. Pak, D. Kim, G. Lee, D. H. Kim, Y. Chung and K. Cho, Adv. Mater., 2016, 28, 5300-5306.

14 Y. Pang, H. Tian, L. Tao, Y. Li, X. Wang, N. Deng, Y. Yang and T.-L. Ren, ACS Appl. Mater. Interfaces, 2016, 8, 26458-26462.

15 C.-L. Choong, M.-B. Shim, B.-S. Lee, S. Jeon, D.-S. Ko, T.-H. Kang, J. Bae, S. H. Lee, K.-E. Byun, J. Im, Y. J. Jeong, C. E. Park, J.-J. Park and U.-I. Chung, Adv. Mater., 2014, 26, 3451-3458.

16 S. G. Lee, H. Kim, H. H. Choi, H. Bong, Y. D. Park, W. H. Lee and K. Cho, Adv. Mater., 2013, 25, 2162-2166.

17 J. Tang, H. Guo, M. Zhao, J. Yang, D. Tsoukalas, B. Zhang, J. Liu, C. Xue and W. Zhang, Sci. Rep., 2015, 5, 16527.

18 D. Rhee, W.-K. Lee and T. W. Odom, Angew. Chem., Int. Ed., 2017, 56, 6523-6527.

19 A. Sabbah, A. Youssef and P. Damman, Appl. Sci., 2016, 6, 152.
20 S. Y. Kim, S. Park, H. W. Park, D. H. Park, Y. Jeong and D. H. Kim, Adv. Mater., 2015, 27, 4178-4185.

21 S. Harada, W. Honda, T. Arie, S. Akita and K. Takei, ACS Nano, 2014, 8, 3921-3927.

22 M. L. Jin, S. Park, Y. Lee, J. H. Lee, J. Chung, J. S. Kim, J.-S. Kim, S. Y. Kim, E. Jee, D. W. Kim, J. W. Chung, S. G. Lee, D. Choi, H.-T. Jung and D. H. Kim, Adv. Mater., 2017, 29, 1605973.

23 X. Liu, Y. Zhu, M. W Nomani, X. Wen, T.-Y. Hsia and G. Koley, J. Micromech. Microeng., 2013, 23, 025022.

24 L. Persano, C. Dagdeviren, Y. Su, Y. Zhang, S. Girardo, D. Pisignano, Y. Huang and J. A. Rogers, Nat. Commun., 2013, 4, 1633.

25 Q. Shao, Z. Niu, M. Hirtz, L. Jiang, Y. Liu, Z. Wang and X. Chen, Small, 2014, 10, 1466-1472.

26 L. Pan, A. Chortos, G. Yu, Y. Wang, S. Isaacson, R. Allen, Y. Shi, R. Dauskardt and Z. Bao, Nat. Commun., 2014, 5, 3002.

27 I. Graz, M. Krause, S. Bauer-Gogonea, S. Bauer, S. P. Lacour, B. Ploss, M. Zirkl, B. Stadlober and S. Wagner, J. Appl. Phys., 2009, 106, 034503.

28 W. Wu, X. Wen and Z. L. Wang, Science, 2013, 340, 952-957.

29 J. Chun, K. Y. Lee, C.-Y. Kang, M. W. Kim, S.-W. Kim and J. M. Baik, Adv. Funct. Mater., 2014, 24, 2038-2043.

30 Q. Sun, D. H. Kim, S. S. Park, N. Y. Lee, Y. Zhang, J. H. Lee, K. Cho and J. H. Cho, Adv. Mater., 2014, 26, 4735-4740.

31 C. Pang, G.-Y. Lee, T.-I. Kim, S. M. Kim, H. N. Kim, S. H. Ahn and K.-Y. Suh, Nat. Mater., 2012, 11, 795-801. 